\title{
Asymptomatic endoalveolar hemorrhage in a young male
}

\author{
Alain Kafyeke, ${ }^{1}$ Giovanni Pomponio, ${ }^{1}$ Andrea Balloni, ${ }^{1}$ Simona Gambini, ${ }^{2}$ Francesca Barbisan, ${ }^{1}$ Armando Gabrielli ${ }^{1}$ \\ ${ }^{1}$ Istituto di Clinica Medica; and ${ }^{2}$ Anatomia Patologica, Dipartimento di Medicina Interna, Università Politecnica delle \\ Marche, Ospedali Riuniti di Ancona, Italy
}

\begin{abstract}
We describe the case of a young male affected by granulomatosis with polyangiitis presenting with non-specific complaints and complicated by the occurrence of a diffuse endoalveolar hemorrhage characterized by atypical clinical and radiological features. The importance of a rapid and aggressive diagnostic and therapeutic approach has to be strongly underlined. Available data regarding prevalence, clinical and radiological characteristics and treatment of this uncommon manifestation have also been hereby reviewed.
\end{abstract}

\section{Introduction}

Granulomatosis with polyangiitis (GP) is an antineutrophil cytoplasmatic (ANCA) associated systemic vasculitis, characterized by necrotizing granulomatous inflammation of small and medium sized vessels. It typically involves the upper airways, lungs and kidney, and more infrequently it affects the gastro-intestine and other organs. Cordier et al. identified the presence of diffuse alveolar hemorrhage in $8 \%$ of patients in their case series. ${ }^{1}$ Acute or subacute onset of dyspnea, cough, and fever are the most common symptoms. The patient can present hemoptysis. This is a severe and potentially lifethreatening manifestation and a prompt and aggressive therapy is recommended.

Granulomatosis with polyangiitis is indeed associated with a considerable morbidity and mortality and, without treatment, approximately $90 \%$ of patients die within 2 years from diagnosis. Therapy involves

Correspondence: Alain Kafyeke and Giovanni Pomponio Istituto di Clinica Medica, Dipartimento di Medicina Interna, Università Politecnica delle Marche, via Conca 10, 60020 Torrette di Ancona (AN), Italy.

Fax: +39.071.5964225

E-mail: alain.kafyeke@gmail.com ;

giovanni.pomponio@ospedaliriuniti.marche.it

Key words: Wegener's granulomatosis; hemorrhage; bronchoalveolar lavage.

Received for publication: 16 February 2015.

Accepted for publication: 21 March 2015.

This work is licensed under a Creative Commons Attribution NonCommercial 4.0 License (CC BY-NC 4.0).

CCopyright A. Kafyeke et al., 2016

Licensee PAGEPress, Italy

Italian Journal of Medicine 2016; 10:137-139

doi:10.4081/itjm.2016.594 aggressive immunosuppression and cyclophosphamide is the first-line drug for inducing remission. Recent evidence also suggests that a treatment with Rituximab is an effective therapy. ${ }^{2}$ However the prognosis directly depends on a timely diagnosis and a swift start of the treatment.

We present an atypical case for which the occasional evidence of an asymptomatic alveolar haemorrhage ultimately led to correct diagnosis and successful aggressive therapy.

\section{Case Report}

A 24-year-old Caucasian man developed during a full month some inflammatory low-back pain and an asymmetric arthritis of wrist and hands associated with low-grade fever and two brief episodes of diffuse abdominal pain. The tests performed during one of these episodes showed mild neutrophil leukocytosis, increased erythrocyte sedimentation rate and Creactive protein, normal liver and kidney function, no abnormalities at urinary standard test. The patient presented no prior risk factors or relevant medical history, except familiarity for psoriasis disease. He was admitted to our hospital for further evaluation. On admission, physical examination did not show any significant alterations. Basing upon the clinical suspicion of psoriatic spondyloarthritis we performed a magnetic resonance image scan of sacroiliac joints, which did not show any signs of sacroiliitis. We also performed a chest X-ray, as part of a pre-treatment screening that revealed an unexpected bilateral, patchy, interstitial pneumonia in the absence of any respiratory symptom (Figure 1A). Lung functional tests showed normal pulmonary volumes and diffusing capacity of the lung for carbon monoxide and a very mild normocapnic hypoxemia. A chest computed tomography (CT) revealed a diffuse perivascular interstitial involvement, with ground-glass and tree- 
in-bud patterns, intralobular consolidations and peribronchovascular micronodules prevalent in declive pulmonary segments (Figure 1B).

We suspected an atypical pneumonia. Blood tests were negative for a viral infection but showed a lowtiter positivity for immunoglobulin (Ig) M and IgA antibodies against Mycoplasma pneumonia, together with an high titer positivity for $\operatorname{IgG}$. Levofloxacin $1000 \mathrm{mg} /$ die was started. During his fourth day at the hospital the patient complained about severe painful paresthesias involving the left median nerve region and the right thumb. An immediate electromyography/ electroneurography of the upper limb confirmed an acute, proximal neuropathy of the left median nerve.

We formulated the hypothesis of a systemic vasculitis and, accordingly, we performed a blood test for auto-antibodies and a bronchoscopy with pulmonary biopsy. The bronchoscopy did not show any mucosal erosion up to the main bronchia. However, the bronchoalveolar lavage fluid was

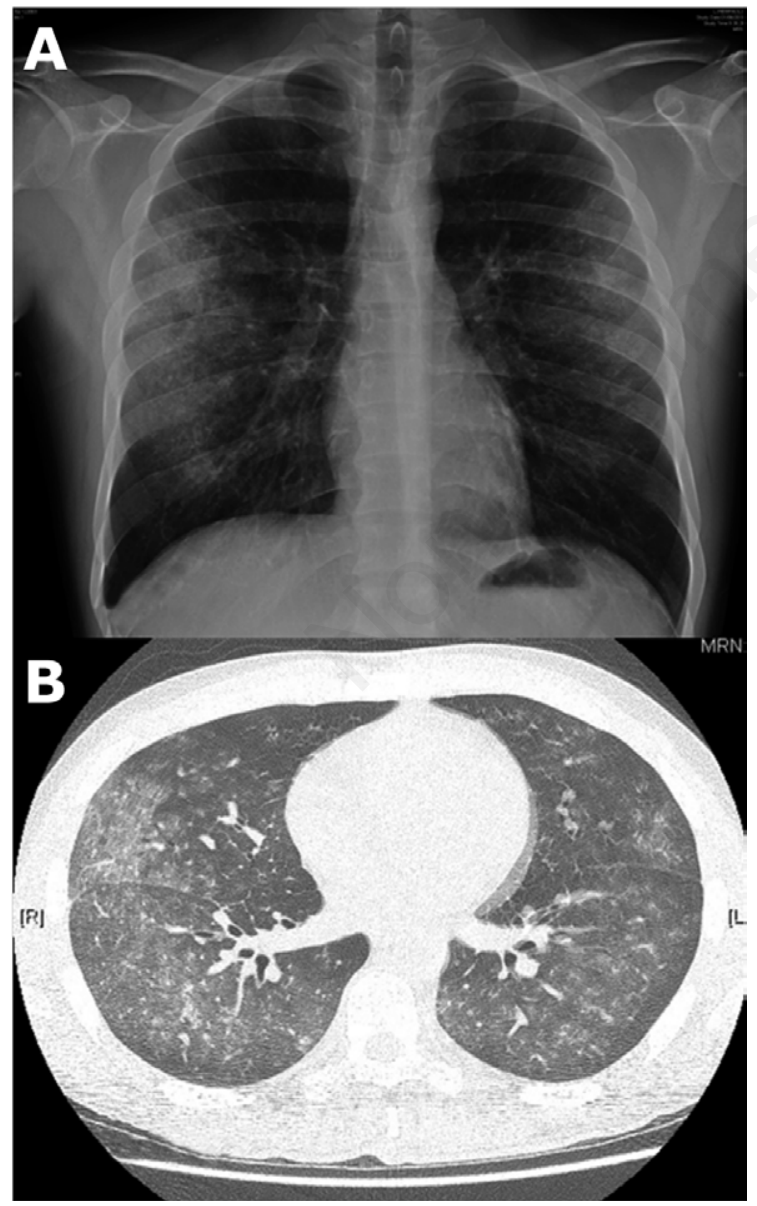

Figure 1. A) Bilateral, patchy, interstitial pneumonia; B) computed tomographic scan showing diffuse perivascular interstitial involvement, intralobular consolidations and peribronchovascular micronodules. hematic (Figure 2A), suggesting an endoalveolar hemorrhage. A specimen obtained via transbronchial lung biopsy from the medial lobe, showed hemosiderin-laden macrophage, erythrocytes, fibrin exudates and neutrophil granulocyte in the alveolar tissue with neutrophilic capillaritis (Figure 2B).

Anti-nuclear antibodies and anti-extractable nuclear antigen antibodies were negative. Antiproteinase 3 antibodies (PR3) were elevated to 191 $\mathrm{UI} / \mathrm{mL}$ (normal values $<2$ ).

Clinical features together with the high titer of anti-PR3 antibodies and the histological findings led
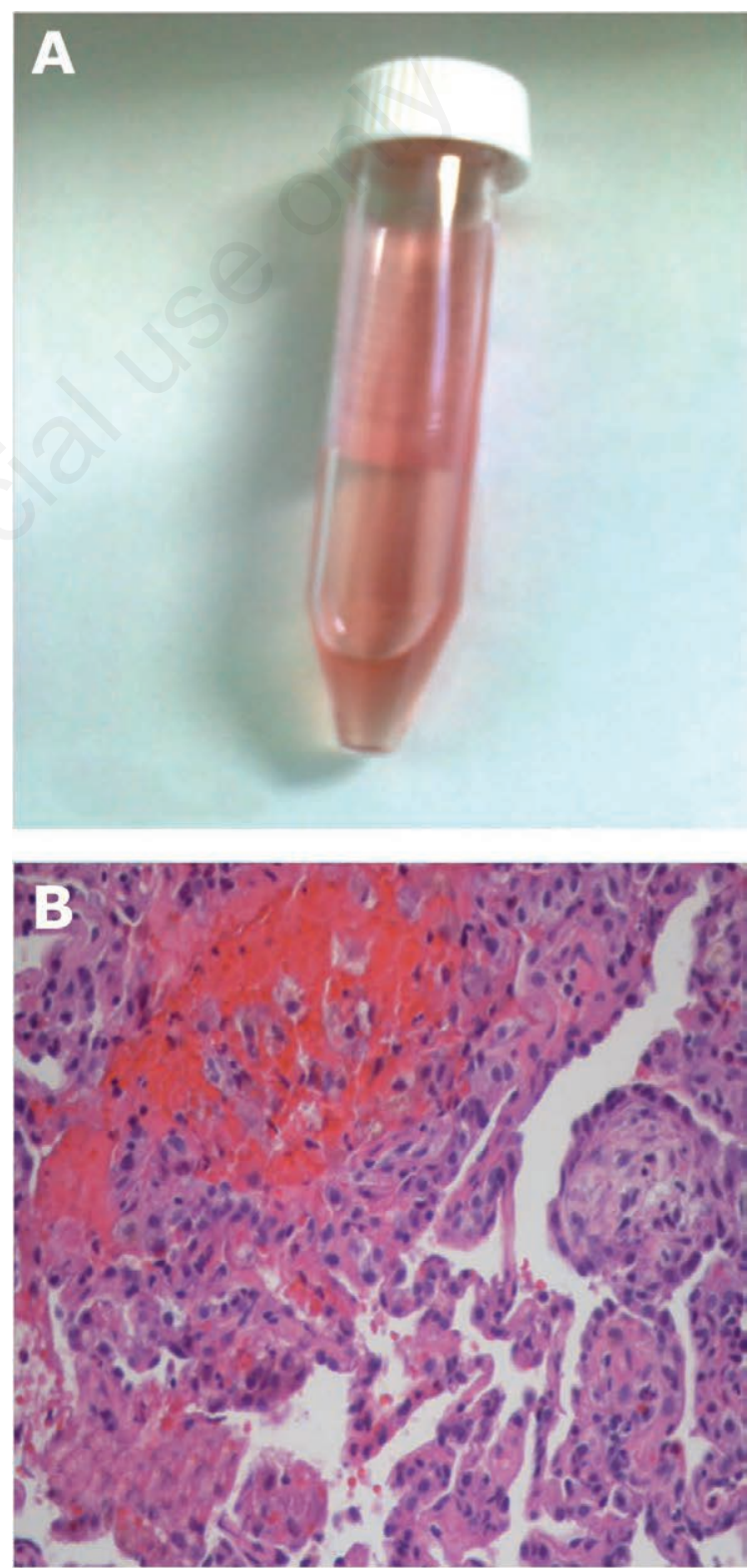

Figure 2. A) Hematic bronchoalveolar lavage fluid; B) pulmonary biopsy showing hemosiderin-laden macrophage, erythrocytes, fibrin exudates and capillaritis. 
to the diagnosis of granulomatosis with polyangiitis. We treated the patient with high dose of methylprednisolone $(1 \mathrm{~g} / \mathrm{die})$ intravenously for three days followed by rituximab $(375 \mathrm{mg} / \mathrm{mq} /$ week for 4 times) in association with oral prednisone, with a prompt clinical and radiological improvement.

A chest $\mathrm{CT}$, performed after two rituximab infusions, showed a complete resolution of the ground-glass consolidations with a reduction in the centrilobular micronodules in the declive pulmonary segments (Figure 3).

\section{Discussion and Conclusions}

Endoalveolar hemorrhage is a well known potentially fatal complication of GP, showing a mortality rate of $60 \%$, six times greater than vasculitis without pulmonary hemorrhage. ${ }^{3}$ A number of diseases can cause a diffuse alveolar hemorrhage and a general classification recognizes cases associated with or without vasculitis/capillaritis or other conditions (infection, malignancy, embolism, etc.).

The clinical presentation can vary from light respiratory symptoms to a severe acute respiratory distress requiring mechanical ventilation. Hemoptysis may be absent because of a large amount of blood being absorbed by the total alveolar volume, ${ }^{4}$ but in a recent evaluation of 80 patients affected by ANCA associated vasculitis presenting with diffuse alveolar hemorrhage, $96.3 \%$ had a history of hemoptysis. ${ }^{5}$

Diffuse ground-glass opacity and consolidation occur in up to $50 \%$ of patients with GP and may result from pulmonary hemorrhage or infection. An extensive ground glass-opacity with subpleural sparing the CT findings is the typical pattern of a diffuse alveolar hemorrhage, but it can only be detected in fewer than $10 \%$ of patients with a pulmonary involvement. ${ }^{6}$

Our case was characterized by clinical and radiological atypical features: in particular, the absence of hemoptysis, dyspnea or cough, the involvement of subpleural spaces, usually spared, the presence of a partial centrilobular distribution and the presence of non-specific enlarged lymph nodes, rarely found in GP. All those elements prevented us to conjecture a diagnosis of vasculitis and made us believe we were facing an infectious disease.

Bronchoalveolar lavage with the evidence of a hematic fluid and transbronchial biopsy were the diagnostic tools that finally led us to the correct diagnosis.

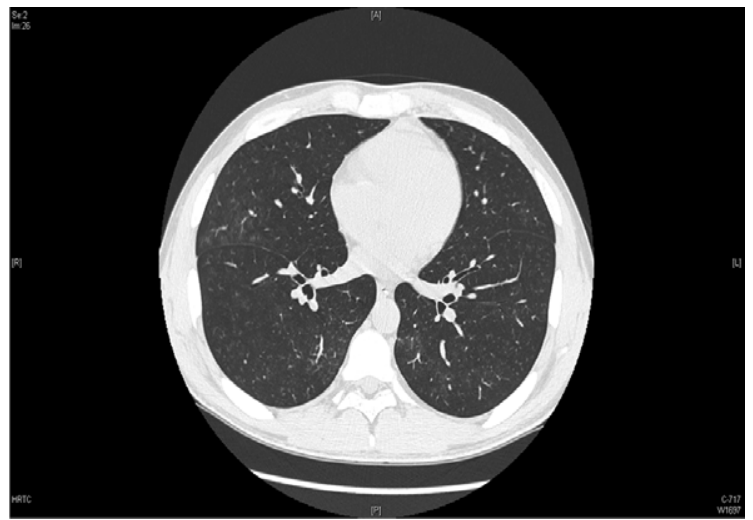

Figure 3. Computed tomographic scan after the second rituximab infusion showing a complete resolution of the ground-glass consolidations and a reduction in the centrilobular micronodules.

In conclusion, in a clinical context compatible with a systemic vasculitis, any evidence of interstitialalveolar damage must redirect to an early and aggressive diagnostic workout, even in the presence of atypical clinical presentation and ambiguous radiological features. This may be essential to a welltimed therapy, able to prevent unfair clinical outcomes.

In our experience the prompt start of the antiCD20 therapy has been effective in dramatically reverse this severe complication.

\section{References}

1. Cordier JF, Valeyre D, Guillevin L, et al. Pulmonary Wegener's granulomatosis. A clinical and imaging study of 77 cases. Chest 1990;97:906-12.

2. Specks U, Merkel PA, Seo P, et al. Efficacy of remission-induction regimens for ANCA-associated vasculitis. N Engl J Med 2013;369:417-27.

3. Green RJ, Ruoss SJ, Kraft SA, et al. Pulmonary capillaritis and alveolar hemorrhage. Update on diagnosis and management. Chest 1996;110:1305-16.

4. Ioachimescu OC, Stoller JK. Diffuse alveolar hemorrhage: diagnosing it and finding the cause. Cleve Clin J Med. 2008;75:258-65.

5. Kostianovsky A, Hauser T, Pagnoux C, et al. Alveolar haemorrhage in ANCA associated vasculitides: 80 patients' features and prognostic factors. Clin Exp Rheumatol 2012;30:S77-82.

6. Martinez F, Chung JH, Digumarthy SR, et al. Common and uncommon manifestations of Wegener granulomatosis at chest CT: radiologic-pathologic correlation. Radiographics 2012;32:51-69. 